DOI: https://doi.org/10.46296/ig.v4i8edespsep.0028

\title{
MODELO LOGÍSTICO DE GESTIÓN DE MANTENIMIENTO COMO ESTRATÉGIA DE MEJORA A LA DISPONIBILIDAD. CASO DE ESTUDIO: UNIDAD DE MANTENIMIENTO DEL GADMEC
}

\section{MAINTENANCE MANAGEMENT LOGISTICS MODEL AS A STRATEGY TO IMPROVE AVAILABILITY. CASE STUDY: GADMEC MAINTENANCE UNIT}

\author{
Solórzano-Calero Emilio Manuel ${ }^{1}$; Espinosa-Delgado Luis Felipe ${ }^{2}$ \\ ${ }^{1}$ Maestrante de la Maestría de Investigación en Mantenimiento Industrial, Mención \\ Gestión Eficiente del Mantenimiento, Instituto de Posgrado, Universidad Técnica de \\ Manabí, UTM. Portoviejo, Ecuador. Correo: esolorzano2286@utm.edu.ec. ORCID ID: \\ https://orcid.org/0000-0001-8594-1966 \\ ${ }^{2}$ Docente de la Carrera de Mecánica de la Facultad de Ciencias Matemáticas, Físicas \\ y Químicas, Universidad Técnica de Manabí, UTM. Portoviejo, Ecuador. Correo: \\ luis.espinosa@utm.edu.ec. ORCID ID: https://orcid.org/0000-0002-7889-7474
}

\section{Resumen}

El modelo de gestión de mantenimiento tiene como objetivo mejorar los índices de disponibilidad operativa aplicando estrategias de monitoreo, control y evaluación con retroalimentación directa en la unidad que proporciona el mantenimiento a los Volquetes Sinotruk del Gobierno Autónomo Descentralizado del Cantón El Carmen (GADMEC), tomando como fuente, el histórico de fallos del sistema y adoptando las particularidades del proceso de mejora continua. La metodología utilizada permite el tratamiento de las variables mediante la ejecución de 5 etapas: definición estrategias del mantenimiento en función del modelo de gestión de mantenimiento basado en el ciclo de Deming que implica 4 pasos: planear, hacer, verificar y actuar (PHVA) propuesto en la norma ISO 9001:2015; la implementación de las estrategias de mantenimiento como herramientas de gestión, el análisis de criticidad de los activos sujetos de estudio, la ejecución de metodología de análisis confiabilidad, mantenibilidad y disponibilidad (CMD) y finalmente, la selección de estrategias de mejoramiento para el manejo recursos. El análisis CMD logró determinar el comportamiento de los indicadores técnicos de gestión de mantenimiento en un periodo determinado de tiempo; los datos obtenidos fueron procesos de forma sistemática y la información resultante permitió medir la incidencia de las estrategias aplicadas, evaluando su comportamiento en función de sus indicadores, estableciendo el crecimiento real de la productividad con la implementación del modelo teórico de gestión de mantenimiento, cuyo enfoque logístico logró la integración entre las unidad de mantenimiento y los activos del Gobierno Autónomo Descentralizado del Cantón El Carmen.

Palabras clave: Disponibilidad operativa, estratégicas, gestión de mantenimiento, confiabilidad, mantenibilidad, disponibilidad.

Abstract

The maintenance management model aims to improve operational availability rates by applying monitoring, control, and evaluation strategies with direct feedback in the unit that provides maintenance to the Sinotruk dump trucks of the Canton El Carmen Autonomous Decentralized Government (GADMEC), taking as a source, the history of system failures and adopting the particularities of the continuous improvement process. The methodology used allows the

Información del manuscrito:

Fecha de recepción: 12 de julio de 2021.

Fecha de aceptación: 03 de septiembre de 2021.

Fecha de publicación: 24 de septiembre de 2021. 
treatment of the variables through the execution of 5 stages: definition of maintenance strategies based on the maintenance management model based on the Deming cycle which involves 4 steps: to plan, to do, to verify and to act (PDCA) proposed in ISO 9001: 2015; the implementation of maintenance strategies as management tools, the criticality analysis of the assets under study, the execution of the reliability, maintainability and availability (CMD) analysis methodology and finally, the selection of improvement strategies for resource management. The CMD analysis was able to determine the behaviour of the maintenance management technical indicators in a given period of time; The data obtained were processes in a systematic way and the resulting information allowed to measure the incidence of the applied strategies, evaluating their behaviour based on their indicators, establishing the real growth of productivity with the implementation of the theoretical model of maintenance management, whose logistics approach achieved the integration between the maintenance units and the GADMEC's assets.

Keywords: Operational availability, strategic, maintenance management, reliability, maintainability, availability.

\section{Introducción}

El implementar estrategias de monitoreo, control y evaluación de las actividades de mantenimiento, son parte fundamental de una gestión y, su incidencia en los activos radica en el aumento de la calidad de prestación de servicios, contemplados en los principios básicos de la mantenibilidad, confiabilidad y disponibilidad, cuya función principal es garantizar el aumento de los niveles de disponibilidad en los equipos $y$ activos físicos de la planta, con el fin de alcanzar los objetivos de la organización mediante una retroalimentación del sistema aplicando el Ciclo Deming, también nombrado ciclo PHVA (Planear Hacer - Verificar - Actuar) como herramienta para la mejora continua en las organizaciones. A partir del análisis de los indicadores de gestión de mantenimiento mencionados, cuya metodología propuso como estrategia la optimización del uso de los recursos enfocados en reducir los costos de mantenimiento mediante la planificación, monitoreo, control y evaluación de las actividades propias de la unidad de encargada de este, se tomó como objeto de estudio a 6 Volquetes Sinotruk del GADMEC.

La disponibilidad es uno de los principales indicadores que se pueden medir en los equipos. Esta permite conocer a detalle, la efectividad de las estrategias implementadas $y$, determinar la incidencia de la propuesta de gestión de mantenimiento adoptada 
por del GADMEC, midiendo el rendimiento de los activos físicos, mediante un análisis comparativo entre el periodo previo a la gestión y los resultados obtenidos a 6 meses de su implementación.

Los estudios de nuevas tendencias en el mantenimiento de las máquinas a nivel mundial recomiendan el uso y aplicación de una amplia gama de técnicas o herramientas de apoyo para esta actividad que buscan la integración dinámica del mantenimiento con la empresa (Shkiliova \& Sanchez, 2011); se estructuró un modelo de gestión de mantenimiento basado en el ciclo PHVA de la ISO 9001:2015 conformado por cuatro factores, que agrupan veinte variables, resultado de la aplicación del análisis factorial exploratorio. Los factores extraídos se catalogaron en las áreas correspondientes a Planificación de Mantenimiento (Planificar), Ejecución del Mantenimiento (Hacer), Medición, Evaluación y Análisis (Verificar) y Actuar (Mejora Continua) (Ortiz Useche, Rodríguez Monroy, \& Izquierdo, 2013).

Estas tendencias en el mantenimiento, hacen énfasis en el tratamiento que se les dé a los activos de una planta, definiendo a esta como un tipo de integración entre los elementos constitutivos de la confiabilidad operacional que permite definir los índices de disponibilidad (Díaz et al., 2021); de aquí que, para lograr un buen entendimiento de las causas de las ineficiencias en cada operación, se tendrá que consultar e involucrar en esta evaluación a los operadores, técnicos y los supervisores (Espinosa, Paz, Pérez, \& Acosta, 2020); y solo en el cálculo de los requerimientos de materias primas $y$ materiales directos consumidos en cada orden de trabajo, llevando un control mediante documentos primarios, relacionando los importes de las materias y materiales directos empleados en las actividades de mantenimiento. (Más, Figueroa, \& Joza, 2019)

Para una correcta aplicación de las estrategias de mantenimiento en los equipos pesados, se debe incluir la Gestión de Ingeniería la administración del Mantenimiento. La primera tiene que ver con la solución técnica de los problemas presentados en las máquinas, implementación de las buenas 
prácticas para la solución de problemas mecánicos o atención de reparaciones, etc. La segunda se encarga de los procesos administrativos de recolección de datos, flujo de la información a fin de tener estos en el momento oportuno, planeamiento y programación, organización adecuada para atender las fallas en el menor tiempo posible, etc. (Zegarra, 2016)

Entre las claves para que los sistemas industriales funcionen de forma eficiente es garantizar la comunicación transversal entre todas las partes interesadas (Mercado \& Peña, 2016); para un mejor manejo del mantenimiento, es necesario realizar el análisis del modo y efecto de fallas (AMEF), comenzado por la parte del diseño, que mejore los resultados en su aplicación y aprovechamiento como metodología a implementar dentro de la gestión del mantenimiento en a los Volquetes Sinotruk del Gobierno Autónomo

Descentralizado del Cantón El Carmen (González, Loyo, López, Pérez, \& Cruz, 2019); se conforma grupos de trabajo que permiten la inspeccionar y diagnosticar, tanto las partes estáticas de los equipos (bastidores y marcos de la maquinaria); así como, las partes dinámicos de las mismos (sistema de frenado, transmisión, dirección, suspensión, etc), para lo cual es necesario contar con el personal necesario para actualizar los períodos de inspección y medición, de acuerdo a los nuevos datos que se obtienen; así como planificar los recursos humanos y materiales (Álvarez \& Hernández, 2020).

Mora (2012) determina que la disponibilidad operacional es la adecuada cuando se requiere vigilar de cerca los tiempos de demoras administrativas o de recursos físicos o humanos; dado que es fundamental trabajar con las actividades planeadas y no planeadas de mantenimiento en forma conjunta, lo cual exige la aplicación de una correcta metodología para su predicción.

Esta disponibilidad (operacional) utiliza los mismos parámetros de la disponibilidad alcanzada, correspondiendo a los tiempos de demoras tales como: tiempos de demora administrativa (ADT), tiempo de inactividad logístico (LDT) y tiempo total logístico que demora 
la acción propia de reparación 0 mantenimiento (LDT').

Con el fin de obtener una base de datos de modos de fallos de los Volquetes Sinotruk del Gobierno Autónomo Descentralizado del Cantón El Carmen (GADMEC), en función del comportamiento de las variables técnicas de cada proceso. Es vital para establecer protocolos y hojas de ruta para la unidad de mantenimiento que enfaticen las actividades y su periodicidad, elaborar una matriz de toma de decisiones que optimicen los indicadores de gestión según se estime necesario. Estas matrices deben ser evaluadas mediante la aplicación de un análisis CMD (confiablidad, mantenibilidad y disponibilidad) para conocer la incidencia de estos indicadores sobre los sistemas más relevantes que componen los elementos sujetos a estudio para buscar mejorar a las actividades de mantenimiento.

La aplicación de un modelo de gestión de mantenimiento basado en el ciclo PHVA de la ISO 9001:2015, logró determinar el tiempo medio entre fallas, el tiempo medio de restablecimiento de la capacidad de trabajo en cada periodo, los índices y el coeficiente disponibilidad; esto genera la necesidad de realizar un análisis de criticidad que permita comprender los rangos relativos a las probabilidades $y / 0$ frecuencias de ocurrencia de eventos y sus consecuencias, sus magnitudes se registran en una matriz (figura 1), diseñada en base a un código de colores que denotan la menor 0 mayor intensidad del riesgo relacionado con la Instalación, Sistema, Equipo o Dispositivo (ISED) bajo análisis (Daquinta, Pérez, Águila, Pérez, \& García, 2018); el uso de las estrategias planeadas en este modelo, permite evaluar el factor confiabilidad de los equipos componentes de los sistemas de las Volquetes Sinotruk del Gobierno Autónomo Descentralizado del Cantón El Carmen (GADMEC), con el uso sistemático de herramientas necesarias para tomar las decisiones adecuadas en razón de las políticas de mantenimiento (Guevara, Valera, \& Gómez, 2015).

Las políticas de mantenimiento establecen protocolos y hojas de rutas que una vez implementadas 
permiten recopilar los datos enunciados en el histórico de fallos a fin de definir la criticidad, el MTBF, MTTR, los índices de mantenibilidad, confiabilidad y disponibilidad operativa. El período de observación se estableció entre los meses de agosto del 2020 y febrero del 2021.

\section{Materiales y métodos}

Al analizar el comportamiento de los activos del objeto de estudio de forma organizada y sistemática se establece un modelo de gestión donde se planifica las actividades en base al estado actual y al estado deseado, mediante el establecimiento de un plan de acción que genere mejoras en las actividades de mantenimiento. Estos procedimientos y sus resultados son evaluados, verificando si las soluciones propuestas tienen el efecto deseado y son satisfactorios para ejecutar la etapa final (actuar) del ciclo PHVA de la ISO 9001:2015 (Tabla 1), donde se debe generar una propuesta de cambio para implementar en el proceso de resolución y dejar sentadas las bases mediante un sistema de mejora continua para las siguientes iteraciones del ciclo.

Tabla 1 Ciclo PHVA.

\begin{tabular}{|c|c|}
\hline CICLO [PHVA] & Actividades \\
\hline Planear & $\begin{array}{l}\text { - Definición de los objetivos de la empresa que sustenten la aplicación de la gestión } \\
\text { - Ide mantenimiento. } \\
\text { mantenimionto de los protocolos necesarios para la aplicación de la gestión de } \\
\text { - Organización las actividades de mantenimiento intrínseco de la planta los } \\
\text { requerimientos legales, sanitarios y ambientales. } \\
\text { - Designación de los recursos a ser utilizados, su tiempo y los términos para su } \\
\text { aplicación. } \\
\text { - Selección de los instrumentos de medición y control de las variables dependiente e } \\
\text { independiente. } \\
\text { - Asignación de funciones y responsabilidades. } \\
\text { - Organización de un histórico de fallos para definir el índice de disponibilidad } \\
\text { operacional. } \\
\text { - Diseño de mecanismos para el control y evaluación de la disponibilidad de los } \\
\text { ítems componentes del sistema. }\end{array}$ \\
\hline Hacer & $\begin{array}{l}\text { - Implantación del diseño metodología de la gestión de mantenimiento que permita } \\
\text { lograr resultados viables en relación los objetivos planteados. } \\
\text { - Evaluación de un proceso de análisis cíclico-sistemático de las estrategias de } \\
\text { mantenimiento. }\end{array}$ \\
\hline Verificar & $\begin{array}{l}\text { - El cumplimiento de las actividades planteadas dentro del diseño metodológico } \\
\text { - Los índices de disponibilidad operativa de los ítems sujetos de estudio }\end{array}$ \\
\hline Actuar & $\begin{array}{l}\text { - Ejecutar las actividades comprendidas dentro de la gestión de mantenimiento con } \\
\text { base en la disponibilidad como aporte al efecto económico al GADMEC. }\end{array}$ \\
\hline
\end{tabular}


Nota: La presente tabla muestra en la metodología PHVA (Planear, Hacer, Verificar y actuar) aplicada en el programa general de investigación para este proyecto.

El tratamiento de los datos generó la información del comportamiento de las mencionadas variables que al ser medidas se pueden contrastar mediante los instrumentos de medición expuestos en el análisis CMD con el empleo de modelos matemáticos que cumplan con los parámetros estadísticos de cálculo de la metodología de análisis de confiabilidad, la mantenibilidad y la disponibilidad, obteniendo la información técnica de los sistemas en función de los tiempos de operación para medir la incidencia de la gestión de mantenimiento con una evaluación integral de los indicadores CMD.

\section{Metodología de análisis CDM}

Las estrategias de mantenimiento implementadas para el manejo y control de los activos de la organización están sustentadas en la información obtenido mediante el procesamiento de la data que permitió generar una metodología acorde para implementar una de gestión de mantenimiento para cada uno de los ítems determinando su criticidad y así, aumentando su productividad de los mismos durante la ejecución de procesos; evidenciada en esta investigación con el costo total en dólares del tratamiento con parte del efecto económico en un periodo de 6 meses. A fin de medir la incidencia de la gestión de mantenimiento aplicada a los Volquetes Sinotruk del GADMEC que pretende alcanzar un aumento de la disponibilidad operativa mediante el control y dirección que ejercen los protocolos y hojas de ruta para la ejecución de las actividades de mantenimiento, la cual establece realizar una evaluación mensual mediante un análisis de los indicadores de confiabilidad, disponibilidad, mantenibilidad aplicando la metodología CMD.

Se estableció un modelo de gestión de mantenimiento preventivo diseñada e implementada para esta investigación, según las necesidades establecidas en evaluaciones previas, en cuyo análisis tomó como referencia los siguientes factores: tiempo operativo, tiempo medio de recuperación de la capacidad de trabajo, el tiempo medio entre fallos, 
el tiempo medio de reparación, confiablidad, mantenibilidad, disponibilidad, disponibilidad inherente o intrínseca, tiempo medio entre mantenimientos, la disponibilidad inherente y la disponibilidad operacional, realizando un seguimiento mensual del comportamiento de los indicadores que sustente la veracidad de los datos procesados comparando los el valor de los indicadores durante los 6 meses antes, con los 6 meses posteriores a la implementación de la gestión de mantenimiento preventivo.

\section{Resultados y discusión}

\section{Criticidad}

La criticidad (grafico 1) muestra el nivel de criticidad en razón de la frecuencia y el nivel de las consecuencias; así, para el sistema de transmisión un nivel de consecuencias de 27, le corresponde un porcentaje de criticidad del sistema es $31,03 \%$; en el sistema de frenado un nivel de consecuencias de 26 y el porcentaje de criticidad del sistema es del 20,69\%; para el sistema de dirección nivel de consecuencias de 17, el porcentaje de criticidad del sistema es del $13,8 \%$ y para el sistema de suspensión, con un nivel de consecuencias de 16, el porcentaje de criticidad del sistema es del $13,79 \%$, convirtiéndolos en los cuatros sistemas más comprometidos y cuya criticidad afecta el funcionamiento de los Volquetes.

Tabla 2. Matriz de criticidad

\begin{tabular}{|c|c|c|c|c|c|c|c|c|c|c|c|}
\hline \multirow{8}{*}{ 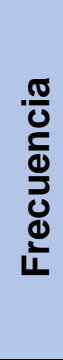 } & 8 & 8 & 16 & 24 & 32 & 40 & 48 & 56 & 72 & 80 & 88 \\
\hline & 7 & 7 & 14 & 21 & 28 & 35 & 42 & 49 & 56 & 63 & 70 \\
\hline & 6 & 6 & 12 & 18 & 24 & 30 & 36 & 42 & 48 & 54 & 60 \\
\hline & 5 & 5 & 10 & 15 & 20 & 25 & 30 & 35 & 40 & 45 & 50 \\
\hline & 4 & 4 & 8 & 12 & 16 & 20 & 24 & 28 & 32 & 36 & 40 \\
\hline & 3 & 3 & 6 & 9 & 12 & 15 & 18 & 21 & 24 & 27 & 30 \\
\hline & 2 & 2 & 4 & 6 & 8 & 10 & 12 & 14 & 16 & 18 & 20 \\
\hline & 1 & 1 & 2 & 3 & 4 & 5 & 6 & 7 & 8 & 9 & 10 \\
\hline & & 1 & 2 & 3 & 4 & 5 & 6 & 7 & 8 & 9 & 10 \\
\hline
\end{tabular}


Nota: La presente grafica muestra la matriz de criticidad de los sistemas componentes de los Volquetes Sinotruk del GADMEC estudiados en base a su frecuencia y el impacto de las consecuencias. Elaboración propia.

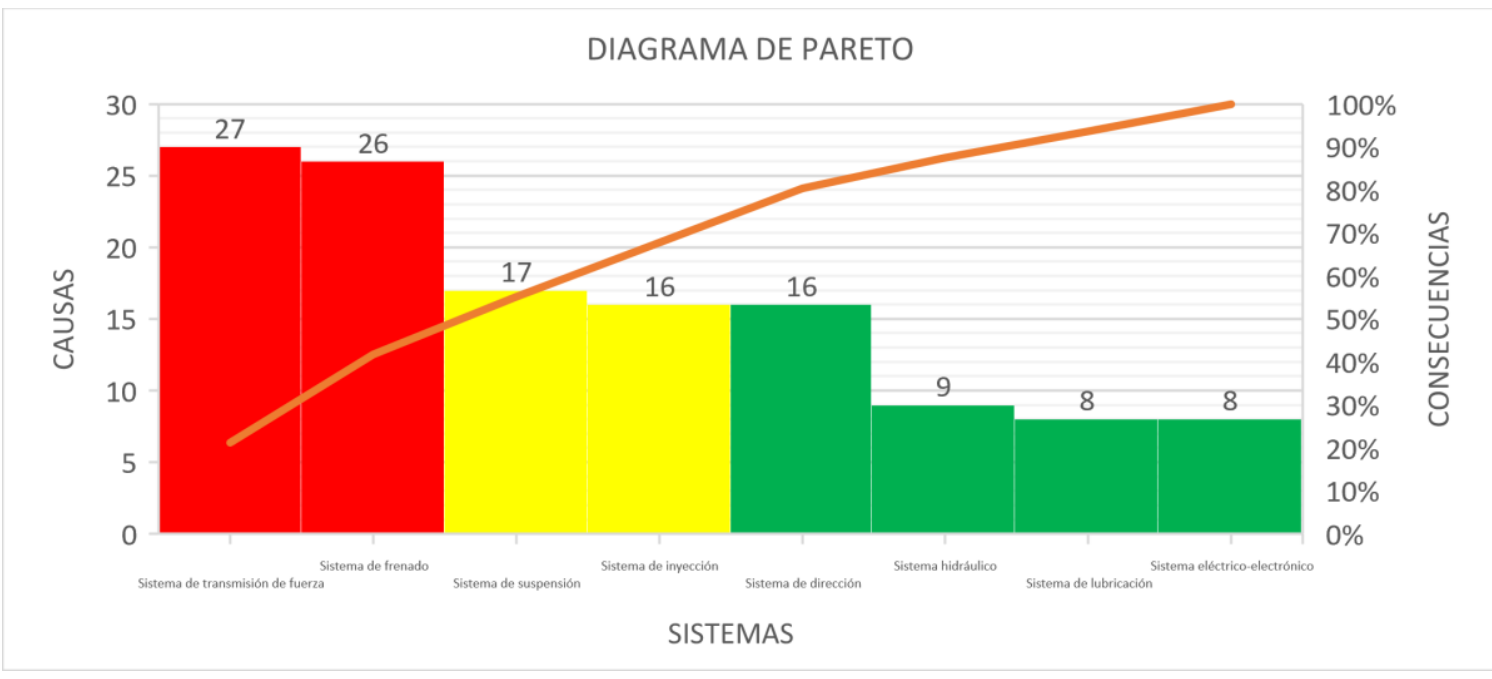

Gráfico 1. Criticidad de los Sistemas, Diagrama de Pareto

Nota: La presente grafica muestra los niveles de criticidad de los sistemas de los Volquetes Sinotruk del GADMEC estudiados en base a su frecuencia y el impacto de las consecuencias. Elaboración propia.

Análisis CMD [Confiablidad, mantenibilidad y disponibilidad]

El Análisis CDM de los sistemas que compara los datos procesados del periodo marzo 2020- agosto del 2020 con el del semestre de septiembre 2020 - febrero 2021 logró determinar los parámetros esenciales del desempeño de los ítems componentes de los sistemas en razón de su confiabilidad, disponibilidad y mantenibilidad de los Volquetes Sinotruk del GADMEC.

Tabla 3. Análisis CMD para los tiempos comparativos entre los semestres marzo a agosto de 2020 y septiembre de 2020 a febrero de 2021.

\begin{tabular}{|c|c|c|}
\hline \multicolumn{3}{|c|}{ TIEMPO MEDIO DE RECUPERACIÓN } \\
\hline Sistema & $\begin{array}{c}\text { Semestre de marzo } \\
\text { a agosto de } \mathbf{2 0 2 0}\end{array}$ & $\begin{array}{c}\text { Semestre de } \\
\text { septiembre de 2020 a } \\
\text { febrero de 2021 }\end{array}$ \\
\hline Sistema de frenado & 20,00 días & 15,00 días \\
\hline $\begin{array}{c}\text { Sistema de } \\
\text { transmisión }\end{array}$ & 22,00 días & 13,00 días \\
\hline Sistema de dirección & 5,50 días & 10,00 días \\
\hline $\begin{array}{c}\text { Sistema de } \\
\text { suspensión }\end{array}$ & 4,50 días & 10,00 días \\
\hline Promedio & $\mathbf{1 3}$ días & $\mathbf{1 2}$ días \\
\hline
\end{tabular}

Nota: La presente tabla muestra los indicadores aplicados al análisis RAM a los Volquetes Sinotruk del GADMEC. Elaboración propia. Elaboración propia 


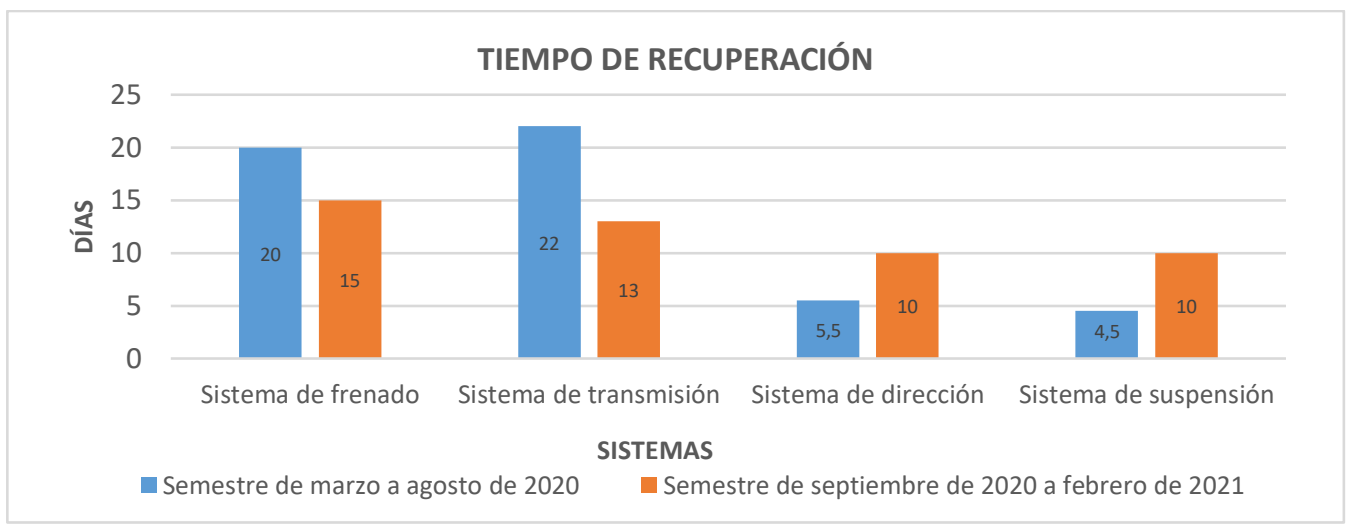

Gráfico 2. Tiempo medio de recuperación.

Nota: La presente grafica muestra el tiempo de recuperación de los sistemas de los Volquetes Sinotruk del GADMEC estudiados en base a su frecuencia y el impacto de las consecuencias. Elaboración propia.

Tabla 4. Análisis CMD para los tiempos medios entre fallos entre los semestres marzo a agosto de 2020 y septiembre de 2020 a febrero de 2021.

\begin{tabular}{|c|c|c|}
\hline \multicolumn{3}{|c|}{ TIEMPO MEDIO ENTRE FALLOS } \\
\hline Sistema & $\begin{array}{c}\text { Semestre de } \\
\text { marzo a agosto de } \\
\mathbf{2 0 2 0}\end{array}$ & $\begin{array}{c}\text { Semestre de } \\
\text { septiembre de 2020 } \\
\text { a febrero de 2021 }\end{array}$ \\
\hline Sistema de frenado & 110,00 días & 120,00 días \\
\hline $\begin{array}{c}\text { Sistema de } \\
\text { transmisión }\end{array}$ & 110,00 días & 122,00 días \\
\hline $\begin{array}{c}\text { Sistema de } \\
\text { dirección }\end{array}$ & 30,00 días & 120,00 días \\
\hline $\begin{array}{c}\text { Sistema de } \\
\text { suspensión }\end{array}$ & 30,00 días & 120,00 días \\
\hline Promedio & $\mathbf{7 0}$ días & $\mathbf{1 2 0 , 5}$ días \\
\hline
\end{tabular}

Nota: La presente tabla muestra los indicadores aplicados al análisis CMD a los Volquetes Sinotruk del GADMEC. Elaboración propia. Elaboración propia.

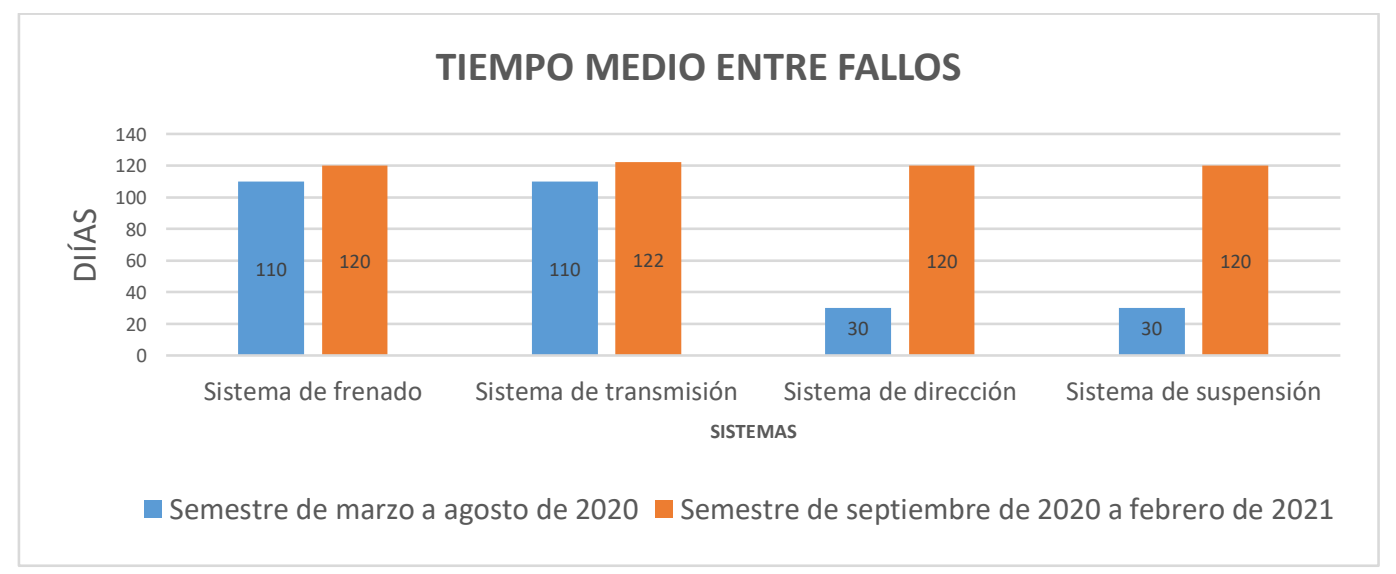

Gráfico 3. Tiempo medio entre fallos

Nota: La presente grafica muestra el tiempo medio entre fallos de sistemas de los Volquetes Sinotruk del GADMEC estudiados en base a su frecuencia y el impacto de las consecuencias. Elaboración propia. 
Tabla 5. Análisis CDM para la confiabilidad entre los semestres marzo a agosto de 2020 y septiembre de 2020 a febrero de 2021.

\begin{tabular}{|c|c|c|}
\hline \multicolumn{3}{|c|}{ CONFIABILIDAD } \\
\hline Sistema & $\begin{array}{c}\text { Semestre de marzo a } \\
\text { agosto de } \mathbf{2 0 2 0}\end{array}$ & $\begin{array}{c}\text { Semestre de septiembre } \\
\text { de 2020 a febrero de 2021 }\end{array}$ \\
\hline Sistema de frenado & $81,87 \%$ & $83,25 \%$ \\
\hline Sistema de transmisión & $81,87 \%$ & $83,50 \%$ \\
\hline Sistema de dirección & $48,03 \%$ & $83,25 \%$ \\
\hline Sistema de suspensión & $48,03 \%$ & $83,25 \%$ \\
\hline Promedio & $\mathbf{6 4 , 9 5 \%}$ & $\mathbf{8 3 , 3 1 \%}$ \\
\hline
\end{tabular}

Nota: La presente tabla muestra los indicadores aplicados al análisis CMD a los Volquetes Sinotruk del GADMEC. Elaboración propia. Elaboración propia.

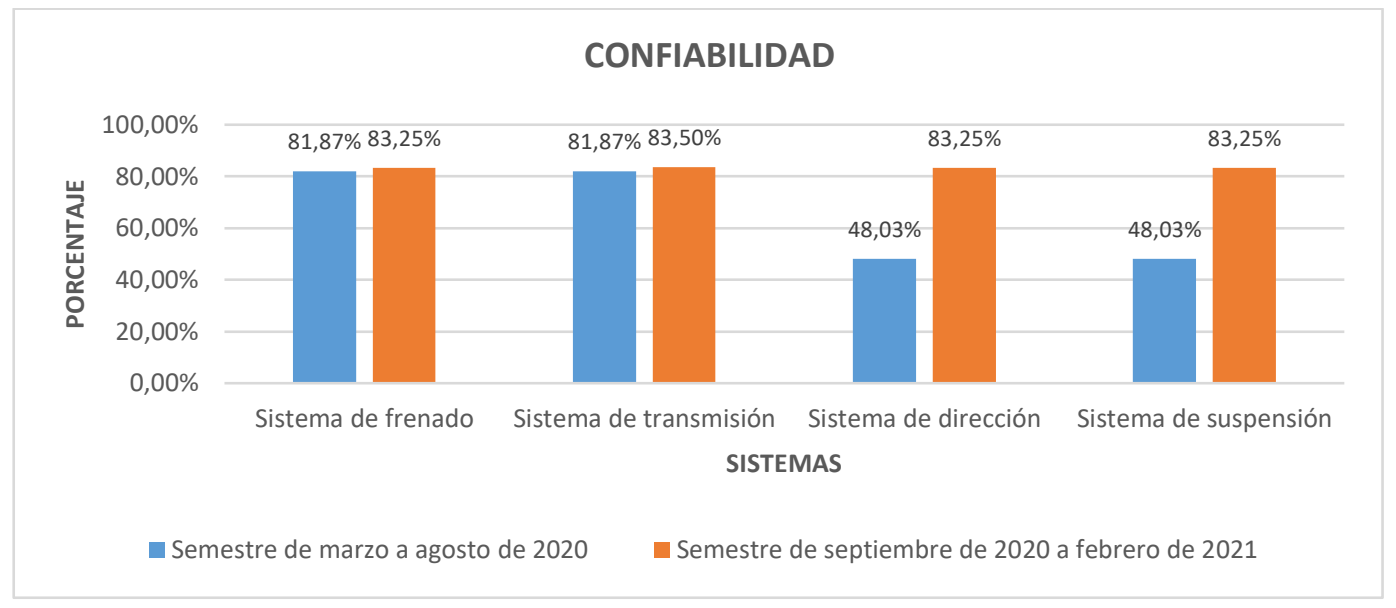

Gráfico 4. Confiabilidad.

Nota: La presente grafica muestra los niveles de confiabilidad de los sistemas de los Volquetes Sinotruk del GADMEC estudiados en base a su frecuencia y el impacto de las consecuencias. Elaboración propia.

Tabla 6. Análisis CDM para la mantenibilidad entre los semestres marzo a agosto de 2020 y septiembre de 2020 a febrero de 2021.

\begin{tabular}{|c|c|c|}
\hline \multicolumn{3}{|c|}{ MANTENIBILIDAD } \\
\hline Sistema & $\begin{array}{c}\text { Semestre de marzo a } \\
\text { agosto de 2020 }\end{array}$ & $\begin{array}{c}\text { Semestre de septiembre } \\
\text { de 2020 a febrero de 2021 }\end{array}$ \\
\hline Sistema de frenado & $86,39 \%$ & $99,98 \%$ \\
\hline Sistema de transmisión & $75,20 \%$ & $99,99 \%$ \\
\hline Sistema de dirección & $100,00 \%$ & $99,99 \%$ \\
\hline Sistema de suspensión & $100,00 \%$ & $99,99 \%$ \\
\hline Promedio & $\mathbf{9 0 , 3 9 \%}$ & $\mathbf{9 9 , 9 9} \%$ \\
\hline
\end{tabular}

Nota: La presente tabla muestra los indicadores aplicados al análisis CMD a los Volquetes Sinotruk del GADMEC. Elaboración propia. Elaboración propia. 


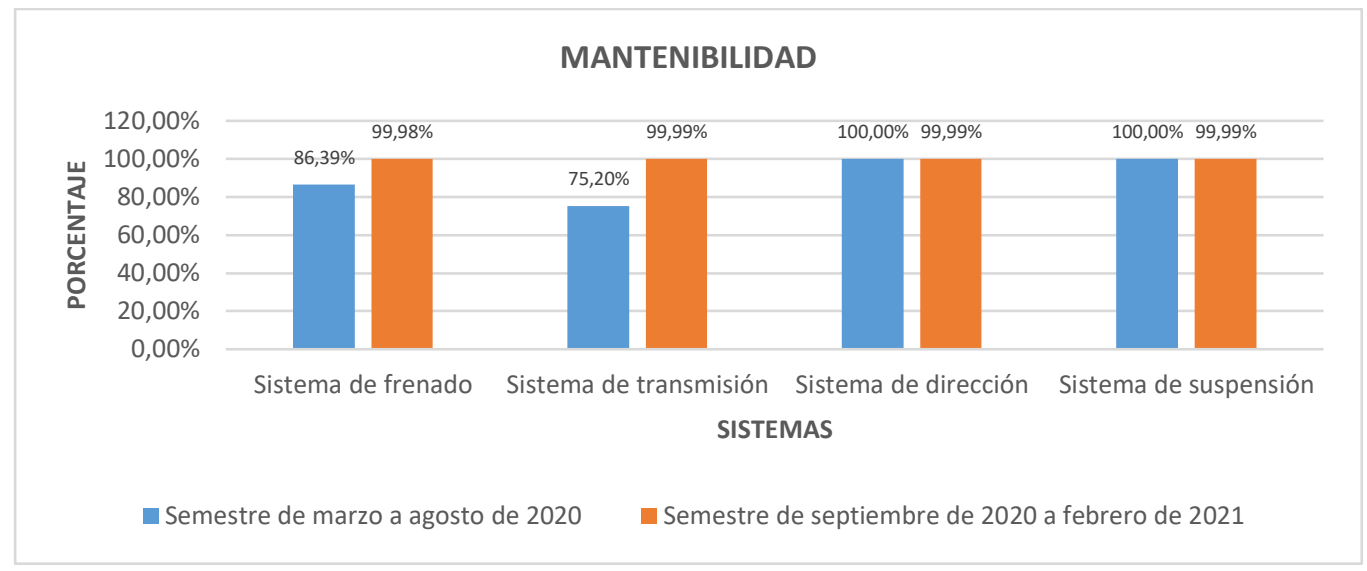

Gráfico 5. Mantenibilidad.

Nota: La presente grafica muestra los niveles de mantenibilidad de los sistemas de los Volquetes Sinotruk del GADMEC estudiados en base a su frecuencia y el impacto de las consecuencias. Elaboración propia.

Tabla 7. Análisis CDM para la disponibilidad general entre los semestres marzo a agosto de 2020 y septiembre de 2020 a febrero de 2021.

\begin{tabular}{|c|c|c|}
\hline \multicolumn{3}{|c|}{ DISPONIBILIDAD } \\
\hline Sistema & $\begin{array}{c}\text { Semestre de marzo a } \\
\text { agosto de 2020 }\end{array}$ & $\begin{array}{c}\text { Semestre de septiembre } \\
\text { de 2020 a febrero de 2021 }\end{array}$ \\
\hline Sistema de frenado & $48,66 \%$ & $90,09 \%$ \\
\hline Sistema de transmisión & $52,13 \%$ & $91,04 \%$ \\
\hline Sistema de dirección & $32,45 \%$ & $92,20 \%$ \\
\hline Sistema de suspensión & $32,45 \%$ & $93,77 \%$ \\
\hline Promedio & $\mathbf{4 1 , 4 2} \%$ & $\mathbf{9 1 , 7 8 \%}$ \\
\hline
\end{tabular}

Nota: La presente tabla muestra los indicadores aplicados al análisis CMD a los Volquetes Sinotruk del GADMEC. Elaboración propia. Elaboración propia.

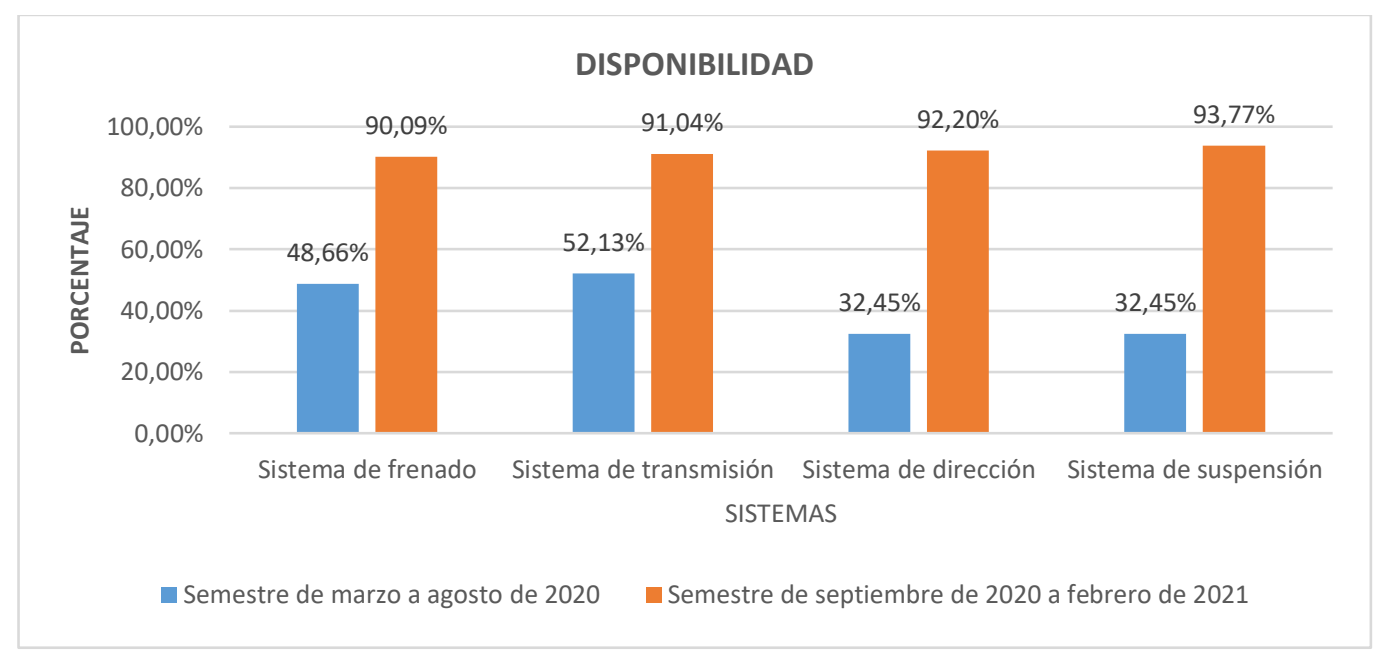

Gráfico 6. Disponibilidad.

Nota: La presente grafica muestra los niveles de Disponibilidad de los sistemas de los Volquetes Sinotruk del GADMEC estudiados en base a su frecuencia y el impacto de las consecuencias. Elaboración propia. 
Tabla 8. Análisis CDM para la disponibilidad inherente entre los semestres marzo a agosto de 2020 y septiembre de 2020 a febrero de 2021.

\begin{tabular}{|c|c|c|}
\hline \multicolumn{3}{|c|}{ DISPONIBILIDAD INHERENTE } \\
\hline Sistema & $\begin{array}{c}\text { Semestre de marzo a } \\
\text { agosto de } \mathbf{2 0 2 0}\end{array}$ & $\begin{array}{c}\text { Semestre de septiembre } \\
\text { de 2020 a febrero de 2021 }\end{array}$ \\
\hline Sistema de frenado & $15,38 \%$ & $11,11 \%$ \\
\hline Sistema de transmisión & $16,67 \%$ & $9,63 \%$ \\
\hline Sistema de dirección & $15,49 \%$ & $7,69 \%$ \\
\hline Sistema de suspensión & $13,04 \%$ & $7,69 \%$ \\
\hline Promedio & $\mathbf{1 5 , 1 5 \%}$ & $\mathbf{9 , 0 3 \%}$ \\
\hline
\end{tabular}

Nota: La presente tabla muestra los indicadores aplicados al análisis CMD a los Volquetes Sinotruk del GADMEC. Elaboración propia. Elaboración propia.

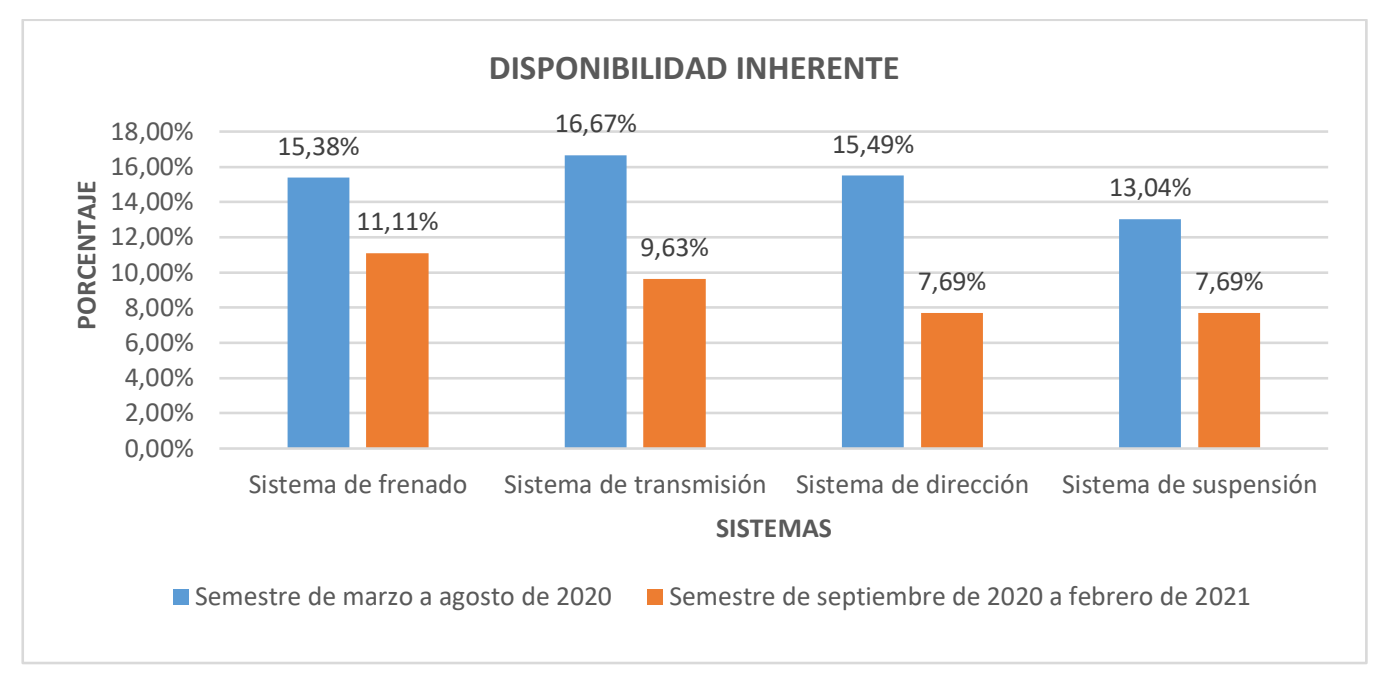

Gráfico 7. Disponibilidad Inherente.

Nota: La presente grafica muestra los niveles de la Disponibilidad Inherente de los sistemas de los Volquetes Sinotruk del GADMEC estudiados en base a su frecuencia y el impacto de las consecuencias. Elaboración propia.

Tabla 9. Análisis CMD para los tiempos medios entre mantenimientos correctivos entre los semestres marzo a agosto de 2020 y septiembre de 2020 a febrero de 2021.

\begin{tabular}{|c|c|c|}
\hline \multicolumn{2}{|c|}{ TIEMPO MEDIO ENTRE MANTENIMIENTOS CORRECTIVOS } \\
\hline Sistema & $\begin{array}{c}\text { Semestre de marzo a } \\
\text { agosto de } \mathbf{2 0 2 0}\end{array}$ & $\begin{array}{c}\text { Semestre de } \\
\text { septiembre de 2020 a } \\
\text { febrero de 2021 }\end{array}$ \\
\hline Sistema de frenado & 70,22 días & 124,33 días \\
\hline $\begin{array}{c}\text { Sistema de } \\
\text { transmisión }\end{array}$ & 74,44 días & 123,67 días \\
\hline Sistema de dirección & 31,67 días & 124,17 días \\
\hline $\begin{array}{c}\text { Sistema de } \\
\text { suspensión }\end{array}$ & 28,75 días & 135,50 días \\
\hline Promedio & $\mathbf{5 1 , 2 7}$ días & $\mathbf{1 2 6 , 9 2}$ días \\
\hline
\end{tabular}

Nota: La presente tabla muestra los indicadores aplicados al análisis CMD a los Volquetes Sinotruk del GADMEC. Elaboración propia. Elaboración propia. 


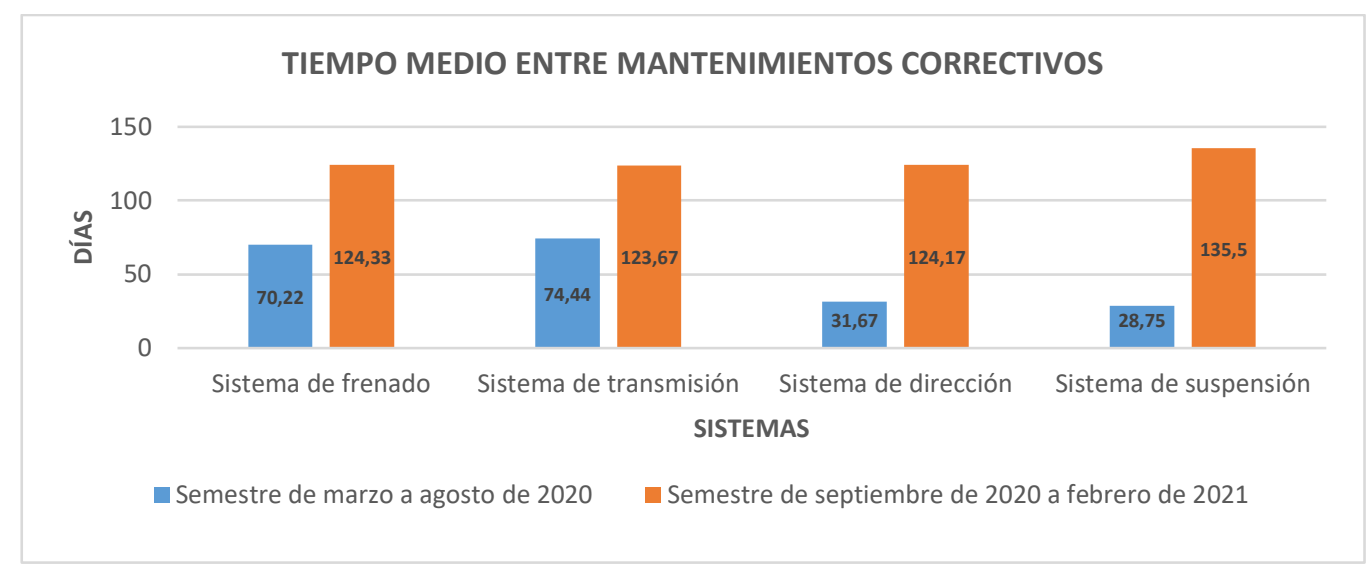

Gráfico 8. Tiempos medios entre mantenimientos correctivos.

Nota: La presente grafica muestra los tiempos medios entre mantenimientos correctivos de los sistemas de los Volquetes Sinotruk del GADMEC estudiados en base a su frecuencia y el impacto de las consecuencias. Elaboración propia.

Tabla 10. Análisis CMD para los tiempos medios entre mantenimientos programados entre los semestres marzo a agosto de 2020 y septiembre de 2020 a febrero de 2021.

\begin{tabular}{|c|c|c|}
\hline \multicolumn{2}{|c|}{ TIEMPOS MEDIOS ENTRE MANTENIMIENTO PROGRAMADOS } \\
\hline Sistema & $\begin{array}{c}\text { Semestre de marzo } \\
\text { a agosto de } \mathbf{2 0 2 0}\end{array}$ & $\begin{array}{c}\text { Semestre de } \\
\text { septiembre de 2020 a } \\
\text { febrero de 2021 }\end{array}$ \\
\hline Sistema de frenado & 52,67 días & 62,17 días \\
\hline $\begin{array}{c}\text { Sistema de } \\
\text { transmisión }\end{array}$ & 55,83 días & 61,83 días \\
\hline Sistema de dirección & 31,67 días & 62,08 días \\
\hline $\begin{array}{c}\text { Sistema de } \\
\text { suspensión }\end{array}$ & 28,75 días & 67,75 días \\
\hline Promedio & $\mathbf{4 2 , 2 3}$ días & $\mathbf{6 3 , 4 6}$ días \\
\hline
\end{tabular}

Nota: La presente tabla muestra los indicadores aplicados al análisis CMD a los Volquetes Sinotruk del GADMEC. Elaboración propia.

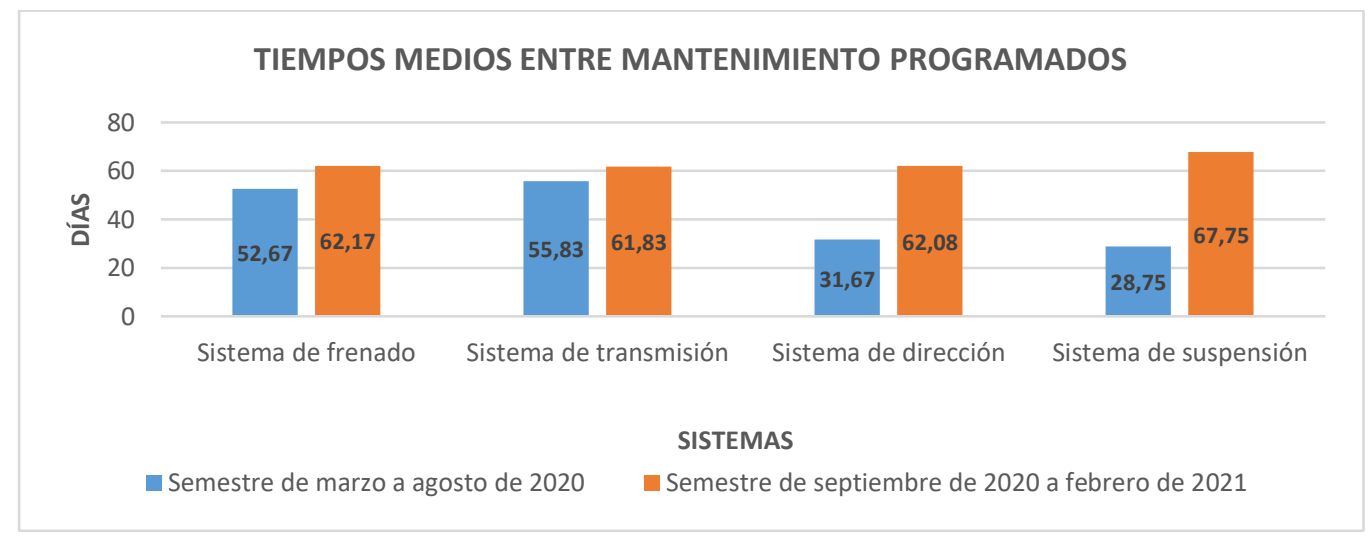

Gráfico 9. Tiempos medios entre mantenimientos programados.

Nota: La presente grafica muestra los tiempos medios entre mantenimientos programados de los sistemas de los Volquetes Sinotruk del GADMEC estudiados en base a su frecuencia y el impacto de las consecuencias. Elaboración propia. 
Tabla 11. Análisis CMD para los tiempos medios entre mantenimientos entre los semestres marzo a agosto de 2020 y septiembre de 2020 a febrero de 2021.

\begin{tabular}{|c|c|c|}
\hline \multicolumn{3}{|c|}{ TIEMPO MEDIO ENTRE MANTENIMIENTOS } \\
\hline Sistema & $\begin{array}{c}\text { Semestre de marzo a } \\
\text { agosto de } \mathbf{2 0 2 0}\end{array}$ & $\begin{array}{c}\text { Semestre de septiembre } \\
\text { de 2020 a febrero de } \\
\mathbf{2 0 2 1}\end{array}$ \\
\hline Sistema de frenado & 30,10 días & 41,44 días \\
\hline $\begin{array}{c}\text { Sistema de } \\
\text { transmisión }\end{array}$ & 31,90 días & 41,22 días \\
\hline Sistema de dirección & 15,83 días & 41,39 días \\
\hline $\begin{array}{c}\text { Sistema de } \\
\text { suspensión }\end{array}$ & 14,38 días & 45,17 días \\
\hline Promedio & $\mathbf{2 3 , 0 5}$ días & $\mathbf{4 2 , 3 1}$ días \\
\hline
\end{tabular}

Nota: La presente tabla muestra los indicadores aplicados al análisis CMD a los Volquetes Sinotruk del GADMEC. Elaboración propia. Elaboración propia.

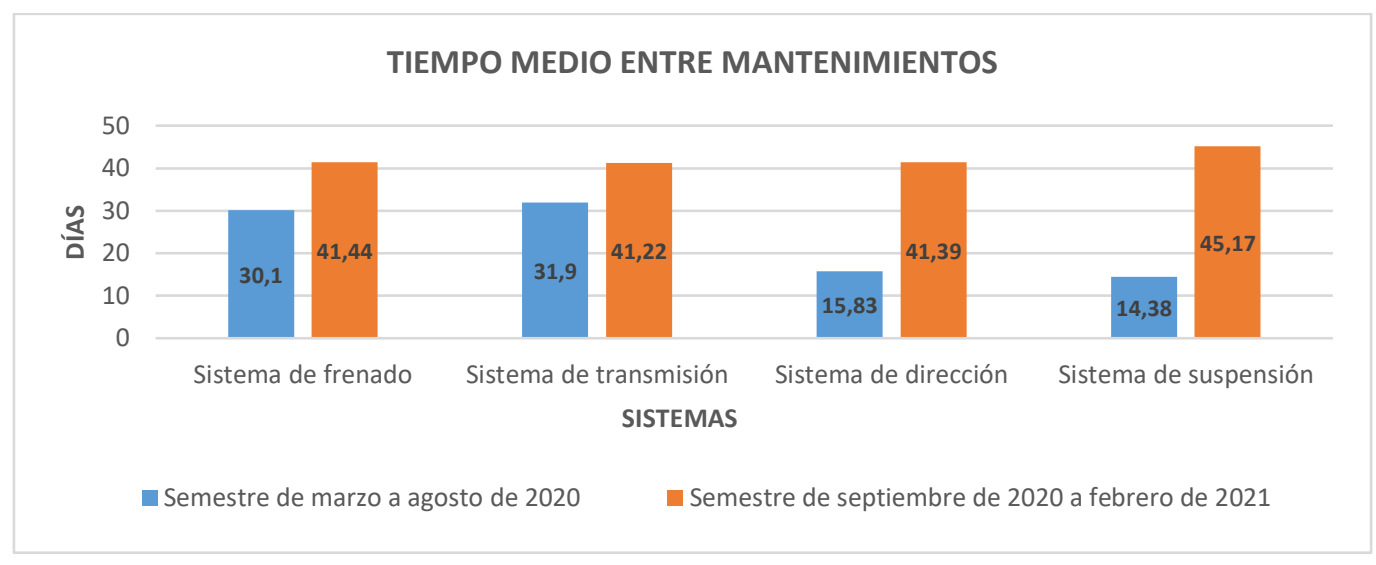

Gráfico 10. Tiempos medios entre mantenimientos generales.

Nota: La presente grafica muestra los tiempos medios entre mantenimientos generales de los sistemas de los Volquetes Sinotruk del GADMEC estudiados en base a su frecuencia y el impacto de las consecuencias. Elaboración propia.

Tabla 12. Análisis CDM para la disponibilidad operativa entre los semestres marzo a agosto de 2020 y septiembre de 2020 a febrero de 2021.

\begin{tabular}{|c|c|c|}
\hline \multicolumn{3}{|c|}{ DISPONIBILIDAD OPERATIVA } \\
\hline Sistema & $\begin{array}{c}\text { Semestre de marzo a } \\
\text { agosto de 2020 }\end{array}$ & $\begin{array}{c}\text { Semestre de septiembre } \\
\text { de 2020 a febrero de 2021 }\end{array}$ \\
\hline Sistema de frenado & 60,08 días & $73,43 \%$ \\
\hline Sistema de transmisión & 59,19 días & $76,02 \%$ \\
\hline Sistema de dirección & 74,22 días & $80,54 \%$ \\
\hline Sistema de suspensión & 76,16 días & $81,87 \%$ \\
\hline Promedio & $\mathbf{6 7 , 4 1} \%$ & $\mathbf{7 7 , 9 7 \%}$ \\
\hline
\end{tabular}

Nota: La presente tabla muestra los indicadores aplicados al análisis CMD a los Volquetes Sinotruk del GADMEC. Elaboración propia. 
Las evaluaciones realizadas a los tiempos operativos y los tiempos de recuperación se realizaron de manera independiente a cada sistema; tomando en cuenta los lineamientos de la metodología de análisis CDM con un enfoque en la mejora continua, jerarquizando los sistemas con mayor criticidad, que permitió conocer su comportamiento.

Este análisis permite conocer cuáles son los tiempos operativos y los tiempos de recuperación que tienen los sistemas tomando como referente los datos obtenidos en este estudio que para el día 180 de la implementación de la gestión aumento los tiempos operativos a una tasa promedio de 98 a 115 horas/mes. Este procesamiento de los datos permitió el cálculo de los índices de MTTR, MTBF, M, R(t), D y $\mathrm{Ai}$, enunciadas de la tabla 4 a la tabla 8; los indicadores de MTBMMC, MTBMMP y MTBM se expresan en la tabla 9 hasta la tabla 11, resultando en el procesamiento estadístico de los datos que se constataron mediante el análisis de los indicadores CMD para los datos procesados del periodo marzo agosto del 2020 con el del semestre de septiembre 2020 - febrero 2021.

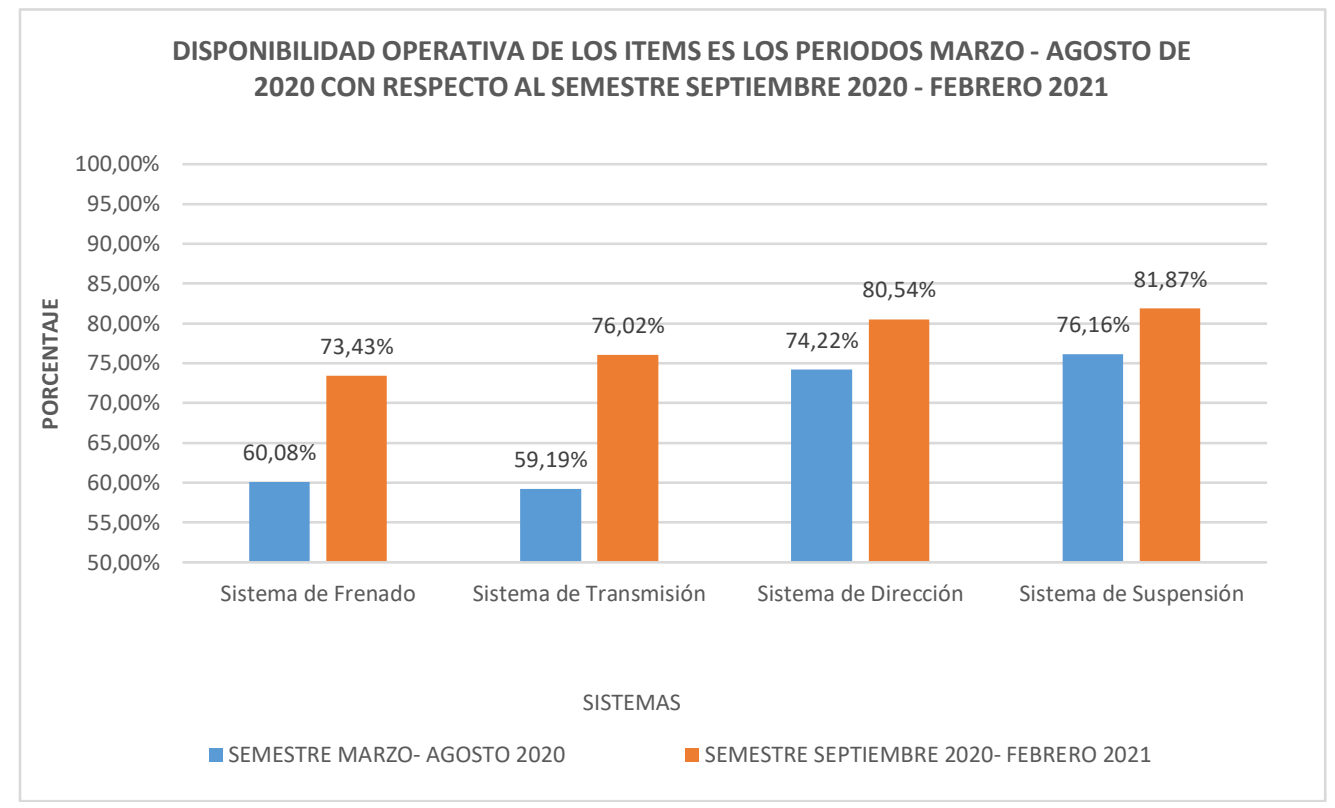

Gráfico 11. Disponibilidad operativa de los ítems previo y posterior a la implementación de la gestión de mantenimiento.

Nota: La presente figura muestra la comparación entre los indicadores de disponibilidad operativa entre los semestres marzo - agosto 2020 y septiembre 2020 - febrero 2021 previo y posterior a la implementación de la gestión integral de mantenimiento a los Volquetes Sinotruk del GADMEC. Elaboración propia. 
El empleo de los protocolos y hojas de ruta como parte de la implementación de la gestión de mantenimiento permite a mejorar la disponibilidad operacional de las 6 Volquetes Sinotruk del GADMEC mediante el control y evaluación de sus actividades aplicando del ciclo PHVA de la ISO 9001:2015 de mejora continua donde una vez realizadas las mediciones, se podrá realizar un reajusta de las expectativas y objetivos predefinidos, para realizar las correcciones y modificaciones necesarias.

\section{Conclusiones}

El comportamiento práctico del modelo de gestión de mantenimiento preventivo para los Volquetes Sinotruk del GADMEC, identifica aumento de los indicadores de confiabilidad, mantenibilidad y disponibilidad; la gestión de los activos fijos (equipos, sistemas, subsistemas, máquinas y herramientas), se corroboran con el análisis CMD realizado a los ítems sujetos de estudios, expresando mejoras en la unidad que se encargó del mantenimiento en es el semestre septiembre 2020 - febrero.
2021, debido a los expuesto en el periodo marzo - agosto 2020.

El modelo de gestión de mantenimiento preventivo diseñado e implementado en esta investigación y basado en el ciclo PHVA de la ISO 9001:2015, demostró la eficiencia en los factores correspondientes a la planificación, ejecución, monitoreo, control y evaluación sistémica del Mantenimiento, mejorando los tiempos medio entre fallos en 50,5 días (tabla 3), los tiempos medios entre mantenimientos correctivos en 75,65 días (tabla 8), los tiempos medios entre mantenimientos programados en 21,23 días (tabla 9) $y$ los tiempos medios entre manteniendo generales en 19,26 días (tabla 10) al redirigir los recursos económicos y técnicos asignados a la unidad encargada de los Volquetes Sinotruk del GADMEC, retroalimentando al sistema y generando una mejora continua.

La evaluación critica de los indicadores de gestión de mantenimiento (confiabilidad, mantenibilidad y disponibilidad) y las aportaciones del modelo la gestión basada en el ciclo PHVA de la ISO 
9001:2015, implantado durante los meses de septiembre del 2020 a febrero del 2021, arrojaron un incremento del $18,36 \%$ en la confiabilidad (tabla 4); 9,6 \% en su mantenibilidad (tabla 5) y el aumento del índice de disponibilidad operativa del objeto de estudio en un $10.56 \%$ (tabla 11). Siendo este el producto de las estrategias de mejoras de organización, planificación y control del mantenimiento.

\section{Bibliografía}

Álvarez, D., \& Hernández, O. (2020). Propuesta de un nuevo programa de mantenimiento a los motores hyundai de grupos fuel oil. Ingeniería Energética, 41.

Daquinta, A., Pérez, C., Águila, J., Pérez, R., \& García, E. (2018). Metodología de Análisis de criticidad integral de las cosechadoras de caña de azúcar CASE IH. Revista Ingeniería Agrícola,, 8, 61.

Díaz, A., Cabrera, J., Castillo, A. d., Benítez, R., Villar, L., \& Rodríguez, A. (2021, 2021). Formulación de un nuevo concepto de confiabilidad operacional/Formulating a new concept of operational reliability. Revista chilena de ingeniería, 49, 87-93.
Espinosa, J., Paz, E. d. I., Pérez, R., \& Acosta, I. (2020). Contribución del mantenimiento centrado en la confiabilidad para el estudio de fallos a equiposconsumidores de energía eléctrica. Centro Azucar, 47, 22-32.

Fernández, M., \& Shkiliova, L. (2012). Validación de un método para el cálculo de indicadores de mantenimiento Validation of a method for the calculation of maintenance indicators. REPARACIÓN $Y$ TECNOLOGÍA MECÁNICA, Vol. 21, No. 4, 72-79.

González, J., Loyo, J., López, M., Pérez, P., \& Cruz, A. (2019). Mantenimiento industrial en máquinas herramientas por medio de AMFE. Ingeniería Industrial, Año 17 №3:, 209225.

Guevara, W., Valera, A., \& Gómez, J. (2015). Metodología para evaluar el factor confiabilidad en la gestión de proyectos de diseño de equipos industriales. Tecnura, 19, 129-141.

ISO, S. C. d. (2015). NORMA INTERNACIONAL ISO 9001: 2015 Sistemas de gestión de la calidad - Requisitos (Vol. 9001: 2015, pp. 45). Ginebra, Suiza.

Más, C., Figueroa, L., \& Joza, M. (2019). Los costos de 
mantenimiento en el taller automotor de la unidad empresarial de base Control de Operaciones en la Empresa de Gases Industriales. Cofin Habana, 14.

Mercado, M., \& Peña, J. (2016). Modelo de gestión de mantenimiento enfocado en la eficiencia y optimización de la energía eléctrica. Revista Multidisciplinaria del Consejo de Investigación de la Universidad de Oriente, 28, 99-105.

Mora, L. (2012). Mantenimiento Industrial Efectivo (Segunda ed.). Medellin, Colombia.

Ortiz Useche, A., Rodríguez Monroy, C., \& Izquierdo, $\mathrm{H}$. (2013, enero-marzo 2013). Gestión de mantenimiento en pymes industriales. Sistema de Información Científica Red de Revistas Científicas de América Latina, el Caribe, España y Portugal Proyecto académico sin fines de lucro, desarrollado bajo la iniciativa de acceso abierto, 18, $N^{\circ} 61$, 86-104.

Shkiliova, L., \& Sanchez, M. F. (2011). Sistemas de Mantenimiento Técnico y Reparaciones y su aplicación en la Agricultura. Revista Ciencias Técnicas Agropecuarias, 20, №1.

Zegarra, M. (2016). Indicators for heavy equipment maintenance management.

Ciencia y Desarrollo. Universidad Alas Peruanas, 19, 25-37. 